\title{
BASICS AND PLANNING
}

nity emergency committee. Joint exercising and testing of the AEP with airport and community responders should occur at frequent intervals.

Conclusion: Airport emergency plans, which dovetail into the community emergency plans, attract a total community response to emergency incidents, which-through its effectiveness-can return the airport to normal operations with minimal delays. AEP can also provide an appropriate response in the treatment of mass casualties.

\section{0}

\section{Medical-Injury Panorama after Different Types of Givilian Aeroplane Accidents \\ Lorin $H$}

National Board of Health and Welfare

Stockholm, Sweden

Objective: With regard to disaster preparedness, it is important to identify what medical resources are needed at both the accident scene and the receiving hospitals after airplane accidents. Methods: Study of the medical consequences from three different types of aircraft accidents with survivors.

Results: The medical-injury panorama is quite different in an airplane accident without fire compared to one with fire. In the first case, if there is a violent crash, there are not many survivors and accordingly rather limited need of medical resources. If there are many survivors, the impact has been comparatively small, and the number of life-threatening injuries low.

If there has been a fire, but no or only a small impact, there can be many victims in need of advanced medical treatment, but evacuation of the victims must be possible. A major problem, other than the burns, is the poisonous gases and heavy, black smoke, which make the evacuation of the injured extremely difficult. These conditions affect the visual, respiratory, and cerebral functions.

\section{6}

\author{
Basics in Disaster Medicine \\ de Boer J
}

Disaster Medicine studies the medical and organization problems of disaster. It is a young branch of medicine and confusion still occurs because relevant terms are used in different ways. The foundation of any science is definition, classification, and measurement. If Disaster Medicine is to grow and progress, it also must have a consistent and recognized definition, classification, and measurement of disasters. By using the criteria "casualties" and "discrepancy between number of casualties and its treatment capacity," a simple definition of a disaster has been formulated. The classification scheme is based on variables which are directly related to disaster, either to its origin or to its effects. By quantifying or weighing these variables and summing the individual scores, a disaster severity scale can be constructed, which runs from 1 to 13 . This approach could provide a firm foundation for the science of disaster medicine, on which basis further development can be confidently expected.

In the event of general international agreement on the definition, classification, and the associated Disaster Severity Scale, it should be possible to assess more accurately, the gravity of a given situation. Additionally, more precise registration would allow scientific comparison of disasters and perhaps also provide an answer to the question of whether the incidence of disaster occurrence is increasing with the growing world population and technology.

A limitation of the practical use of this scoring system is that it can only be applied retrospectively. For this reason, the medical severity index has been introduced. This index not only indicates the breaking point between accident and disaster, but also quantifies the medical severity instantaneously.

Moreover, the index can be used in the disaster preparedness phase for estimating the requirements needed to produce a desirable capacity within the various links in the chain of medical care from the disaster site to the hospital bed.

The methodologies used are described.

17

\section{Why Disaster Medicine?}

Debacker $M$

Medical Service Belgian Armed Forces

Brussels, Belgium

The chances that medical and paramedical personnel will be confronted with the effects of disasters rise as the world population grows and as technological and social developments increase. There also are indications that the number of natural disasters is increasing and that this change is possibly linked to human-made environmental changes. The objectives of Disaster Medicine are to prevent, reduce, and mitigate the effects of disasters on the health of affected populations and to restore, as soon as possible, health services and facilities to the pre-disaster situation. A different medical approach is necessary to 
achieve these goals due to the immediate effects of the disaster on the community and especially on the health care system, to the number and variety of casualties, to the emergency situation with an initial phase of disorder, to the lack of resources and the limited output of medical teams, to the necessity to operate in multidisciplinary and complementary teams and to the multiplicity of tasks. Therefore, Disaster Medicine has its own characteristics-it is an emergency medicine in the field, a global medicine, a mass medicine, a medicine with extramedical aspects, and a doctrinal medicine. Therefore, education and training in Disaster Medicine are essential.

\section{8}

\section{The Link Between Emergency, Disaster, and War Medicine}

Beerstecher $H$

Chairman, Joint Medical Committee, NATO

Brussels, Belgium

Objective: To inform the congress inter alia of the activities of the Joint Medical Committee of the North Atlantic Treaty Organization.

Methods: The scope of Emergency, Disaster, and War Medicine is outlined. From the medical point of view, Emergency Medicine is the common denominator of Disaster and War Medicine and, therefore, is a line of itself.

With the exception of some essential differences, a lot of similarities between Disaster and War Medicine are evident. It was stated in the past that emergency medical care in disasters should follow the principles of emergency war surgery (Spirgi, 1979). Therefore, it is concluded that Emergency and War Medicine are the pillars on which Disaster Medicine is built.

From the managerial point of view, links can be identified between the military-medical and civil-defense organizations on local, national, and international governmental levels. In this context, some activities in the field of medical and health care within the organization of the North Atlantic Alliance are outlined.

A relation which might possibly evolve between NATO and the United Nations Department of Humanitarian Affairs (DHA-GENEVA) is mentioned.

Conclusion: In order to avoid duplication of effort and to make full use of each others' possibilities, it is concluded that military and civil-defense assets should also be used in disaster relief.

\section{9}

\section{Changing Concepts and Perspectives in Disaster Medicine}

Brismar B

Soder Hospital, Karolinska Institute

Stockholm, Sweden

During the last decade, the medical care systems in the United States and Europe have been characterized by an ever increasing shortage of money. The need for economical measures have become pronounced and market economy principles have been introduced. The scene now is dominated by purchasers and providers, competition between public and private enterprises, and economical considerations guide the offer of medical treatment.

The need for extensive rationing with maximal utilization of resources such as personnel, localities, and supply, has resulted in increased vulnerability. Only a limited extra load on the medical services, e.g., after a major accident, results in prioritization of problems. The possibilities to cope with a disaster situation with a substantial number of casualties vanishes when all the margins already are being used and no more capacity can be mobilized. In such a situation, the knowledge and experience gained from Disaster Medicine are more important than ever before. Well-founded, cost-effective analysis will be essential for the medical care system to be able to handle the limited resources properly, and to set the right priorities. Which treatment principles should be practiced to obtain the best possible results for as many patients as possible under safe conditions? How small are acceptable margins? These are questions that need answers. Conclusion: It will be the responsibility of experts in Disaster Medicine to take a more active part in this discussion and to present solutions acceptable from an ethical as well as economical standpoint.

\section{0}

\section{The Role of Telemedicine in the Management of Disasters}

Manni $C$

Director of Anaesthesiology and Intensive Care

Catholic University School of Medicine

Rome, Italy

A disaster represents a real challenge for the medical and social structures; it may stress greatly the response capability of both the emergency medical and rescue services. Although it virtually is impossible to completely remove the risk of disaster, many efforts should be made in order to prevent and limit its consequences. Effective management of disasters requires accurate preventive planning and cooperation between all involved structures, including: Police Force; Armed Forces; Emergency Departments; Poison Control Centers; hospitals; and rescue staffs. From this point of view, telemedicine, a new and rapidly developing field of medical sciences, is now opening interesting perspectives. Many telemedicine applications, such as teleconsultation and tele-electrocardiography, allow long distance data communication between health care professionals for diagnostic and therapeutic planning. The development of a computerized data bank with a mass storage system offers quick access to data concerning the disaster risk in selected areas, the most important clinical information for patient management and the availability of specific health care facilities. Continuous up-to-date and real-time information is particularly important in toxicological emergencies due to the large number of toxic substances that may be involved, and the many different mechanisms of action by which they exert their activities. The preparedness of the single health care professional is not sufficient to handle the complex reality of a disaster. The full integration of the emergency medical system is 